\title{
Visual Association Rules on the Psychological Connection of University Students with their Studies
}

\author{
Erika Yunuen Morales Mateos ${ }^{1}$, María Arely López Garrido ${ }^{1}$, \\ José Alberto Hernández Aguilar ${ }^{2}$, Carlos Alberto Ochoa Ortiz ${ }^{3}$, \\ Oscar Alberto González González ${ }^{1}$, Arturo Corona Ferreira ${ }^{1}$ \\ ${ }^{1}$ Universidad Juárez Autónoma de Tabasco Cunduacán, Tabasco, Mexico \\ ${ }^{2}$ Universidad Autónoma del Estado de Morelos, Cuernavaca, Morelos, Mexico \\ ${ }^{3}$ Universidad Autónoma de Ciudad Juárez, Ciudad Juárez, Chihuahua, Mexico \\ erika.morales@ujat.mx, arely.lopez@ujat.mx, \\ jose hernandez@uaem.mx, alberto.ochoa@uacj.mx, \\ oscar.gonzalez@ujat.mx, arturo.corona@ujat.mx
}

\begin{abstract}
The objective of this work is to express patterns of data behavior related to the psychological connection of the university students with their studies, this through the association rules, likewise these rules are presented in an interactive visual way, which facilitates the review of these to select those that are considered of interest. For this study, students of four careers in technologies from a university in southern Mexico were considered. To measure the psychological engagement with the studies, the Utretch Work Engagement Scale for Students instrument (UWES-S) was used, other elements were included to know opinions related to careers. The learning algorithm of association rules A priori was used. For the generation and interactive visualization of the association rules, the Apriori algorithm was used in the R language. It is concluded that the interactive visual display is an important support for the selection of the rules that are considered relevant.
\end{abstract}

Keywords: rules of association, visualization, apriori, psychological connection, student engagement.

\section{$1 \quad$ Introduction}

Research is making an important advance given that nowadays it is possible to rely on techniques that offer different forms of data analysis. The data storage to subsequently apply different forms of knowledge extraction has presented a great advantage, which can be applied to the development of psychological, educational, and other studies. Many of the psychological studies usually involve applying questionnaires, which can be automated to generate data sets that include these items, for further analysis. Data mining techniques offer several options for discovering knowledge, and it is possible to make use of them in psychology.

One area of psychology where much research is carried out is that of occupational health, which studies syndromes such as burnout that determines to what extent people 
feel physically and mentally exhausted, or the engagement to determine human potential and psychological capacities in his activities such as work or professional studies. The engagement in the activities is defined as "engagement is a positive psychological state characterized by high levels of energy and vigor, dedication and enthusiasm for work, as well as total absorption and concentration in the work activity" [1]. This concept of engagement has also been taken to the universities, defining it as the psychological engagement with the studies. These data allow knowing new factors that impact on the training of university students, their dedication, absorption and vigor, in relation to the studies. The Utrecht Work Engagement Scale Students (UWES-S) [2] is used to know the levels of the dimensions that involve the psychological connection with the studies.

As mentioned from the data generated from psychological instruments, the application of data mining is possible. Data mining aims to analyze the data to extract knowledge. The knowledge obtained can be in the form of relationships, patterns or rules inferred from the data, or in a more concise description. These relationships or summaries integrate the model of the data analyzed. The models can be represented in different ways and each of them determines the type of technique that can be used to infer them. Descriptive models identify patterns that explain or summarize the data, work to explore the properties of the data examined, but do not predict [3]. Association rules are a descriptive task, similar to correlations that identify non-explicit relationships between categorical attributes. There can be many formulations, the most common is the one of the style: if the tribute $\mathrm{X}$ has the value to then the attribute $\mathrm{Y}$ has the value $b$. These rules do not involve a cause-effect relationship, so there may be no cause for the data to be related. The rules are evaluated using the precision and support parameters [3].

The presentation of data by visual techniques such as the rules of visual association is that they help to understand the behavior of data with a glance, in this way data analysts can save time when making judgments about patterns, trends, variability, among others, of the data. One of the advantages of using visual techniques to represent data is that they can show characteristics that would otherwise have been complex or impossible [4].

For the development of type of studies that involve generating graphics such as visual association rules, the $\mathrm{R}$ language is suggested, which is an integrated collection of software services for data management, calculation and visualizations. $\mathrm{R}$ is free under the terms of the GNU General Public License of the Free Software Foundation [5].

In this work, we propose to know the psychological engagement with the university studies, as well as other variables related to their career, which imply in the decision of stay and continuity of the studies, for this purpose it is proposed to use a data mining task: The Association Rules. These rules let to express patterns of a data set, these patterns allow to know the general behavior of the data and in this way obtain descriptive information that can assist in decision making. Likewise, it is intended to go a step further, presenting the resulting rules in a visual graphic format, which facilitates the analysis and selection of the rules of interest.

There are works in which educational and psychological studies have been carried out, where data mining techniques are used, this is the case of the data mining work 
applied to the identification of risk factors in students in the state of Mexico, among the techniques used is that of association rules [6], a model was proposed for school desertion in Universities of Mexico [7], on the same subject in terms of school failure prediction [8], another work was developed to evaluate parameters of the CENEVAL entrance survey for students who are candidates to enter the upper level, an ITP study case, for which a classification model was generated [9]. There are also works where visual association rules have been generated for different administrative problems, natural disasters, among others [10,11]. Additional work on this research was developed using data analysis techniques based on graphic representation $[12,13]$.

\section{$2 \quad$ Materials and Proposed Methods}

\subsection{Description of the Data}

The objective of this research is to present the results obtained from the application of the rules of association under the Apriori algorithm, including its interactive visualization, to a set of data containing information about psychological engagement, obtained by applying the UWES-S instrument, and other variables related to his career, which imply in the decision of stay and continuity of the studies. The students belong to a sample population of four computer science degrees in a computer and systems faculty, in a university in southern Mexico. The bachelor's degrees considered for this study were the Computer Systems (LSC), Information Technology (LIA Information Technology (LTI) and Telematics (LT). The sample was non-probabilistic, directed and by convenience [5], a survey was applied to a total of 141 students, who accepted to answer the questionnaire voluntarily, in the period February-August 2015 carried out the data collection.

\subsection{Wellness Scale in the Academic Context (Utrecht Work Engagement Scale for Students, UWES-S)}

The psychological connection with the studies was obtained with the application of the Utretch Work Engagement Scale for Students instrument (UWES-S). This instrument has its origins in the need to know the psychological connection with the activities carried out at work, that is, the engagement to work. Thus, the engagement to work was defined by Salanova and Schaufeli [1] as: "a positive psychological state characterized by high levels of energy and vigor, dedication and enthusiasm for work, as well as total absorption and concentration in the work activity". To measure this concept Schaufeli and Bakker [2] developed the UWES-S, this instrument was subsequently modified, giving rise to a version that would allow knowing the psychological engagement with the studies or also known as engagement to the studies, because the studies are considered an activity in which they have responsibilities and a goal, just like the work. In this version of the instrument for students the same concepts and instruments of engagement were used in the work, there are some differences in the writing of the elements adapting them to the student activity, giving rise to the questionnaire Utretch Work Engagement Scale for Students (UWES-S) [2]. This UWES-S consists of 17 items, divided 
into three constructs, vigor, dedication and absorption. The vigor consists of six items, which refer to high levels of energy and resilience, willingness to dedicate efforts, not fatigue easily, and persistence in the face of difficulties. The dedication, composed of five items that refer to the meaning or meaning of the work, to feel excited and proud of their work, and feel inspired and challenged by the work. Absorption, which consists of six items that refer to being happily immersed in your work and present difficulty in leaving it, so that time passes quickly, and you forget everything around you "[2].

In the UWES-S the answers are measured according to a Likert scale, where zero means "never" and six "always", in this way the scores go from zero to six for each dimension that makes up the student engagement. The original interna consistency obtained by the authors for the UWES-S version of 17 items in Dutch students was for strength of 0.63 , dedication 0.81 and absorption of 0.72 , complying with the criterion of superiority to 0.60 for an instrument of recent development. The specifications of the instrument can be found in the manual of Schaufeli and Bakker [2].

\subsection{Analysis of Data}

The generation of association rules was carried out based on the Apriori algorithm, to find behavior patterns between the data according to the joint appearance of values of two or more attributes, as well as the interactive visual display of these rules to facilitate the observation and selection of the rules. To generate these results, the $\mathrm{R}$ language was used, since it is a powerful and versatile software, especially in graphics development, because it has packages that allow complex analysis and design custom graphics, as well as programming at an advanced level [14].

\section{Association Rules}

An association rule is a correspondence between itemsets, an itemset is a set of one or more items. If $\mathrm{X}$ and $\mathrm{Y}$ are two itemsets, the rule that associates $\mathrm{X}$ with $\mathrm{Y}$ is written: $\mathrm{X}$ $\rightarrow \mathrm{Y}$, where $\mathrm{X}$ is the antecedent of the rule and $\mathrm{Y}$ the consequent. There are two measures for evaluating the support of an association rule and the confidence of an association rule [15]:

- The support of an association rule is defined as the proportion of transactions that contain the antecedent and the consequent of the rule.

$$
\operatorname{supp}(\mathrm{X} \rightarrow \mathrm{Y})=\operatorname{supp}(\mathrm{X} \cup \mathrm{Y}) \text {. }
$$

- The confidence of an association rule is defined as the proportion of transactions that are met when the rule can be applied.

$$
\operatorname{conf}(\mathrm{X} \rightarrow \mathrm{Y})=\operatorname{supp}(\mathrm{X} \cup \mathrm{Y}) / \operatorname{supp}(\mathrm{X}) \text {. }
$$

It is important to note that the confidence of the rule $\mathrm{X} \rightarrow \mathrm{Y}$ is an estimate of the conditional probability of $\mathrm{Y}$ given $\mathrm{X}$ obtained from the relative frequencies of occurrence of $\mathrm{X}$ and $\mathrm{Y}$. The results of an association analysis in a particular application must be taken with caution. The fact that a rule $\mathrm{X} \rightarrow \mathrm{Y}$ has a high confidence does not directly result in $\mathrm{X}$ being the cause of $\mathrm{Y}$. The rule shows the simultaneous occurrence of $\mathrm{X}$ and $\mathrm{Y}$, but not that $\mathrm{X}$ is the cause and $\mathrm{Y}$ the consequence. 
The application of the association rules is described: Given a universe $\mathrm{U}$, a set of transactions $\mathrm{T}$ and two values minsupp $\in[0,1]$, minconf $\in[0,1]$ find all the association rules $\mathrm{X} \rightarrow \mathrm{Y}$ that comply with the following two elements [15]:

- Minimum support supp $(\mathrm{X} \rightarrow \mathrm{Y}) \geq \operatorname{minsupp,}$

- Confidence minima conf $(\mathrm{X} \rightarrow \mathrm{Y}) \geq$ minconf.

An exhaustive search is carried out where all the association rules that can be obtained from universe $U$ must be generated, calculate the support and trust of each rule taking into account the set of transactions $\mathrm{T}$, store the rules that exceed the minimum thresholds minsupp and minconf

If the set has k elements, the number of rules that can be obtained is $2 \mathrm{k}-2$. The search for rules that meet the minimum support and trust requirements can be developed in two stages [15]:

- Generate frequent sets. Find the sets of items whose support is greater than or equal to minsupp.

- Build the association rules. For each of the sets obtained in the previous stage, find the association rules that have a confidence greater than or equal to minconf.

For the efficient generation of frequent sets it is important to consider that if a set of items is not frequent, neither will the sets that contain it. This is how the search tree is pruned and the efficiency of generating frequent sets is increased.

The measure of statistical independence lift is defined as a relationship between the simultaneous occurrence of $X$ and $Y$, when the sets of items that make up the antecedent and the consequent of the rule are statistically independent. Experts in the area conclude that knowledge rules discovered through support and trust should be filtered using their lift values, since lift values greater than 1 indicate association between items; and values less than 1 indicate their independence between items and should not be considered for decision making [16]:

$$
\operatorname{lift}(\mathrm{X} \rightarrow \mathrm{Y})=\operatorname{conf}(\mathrm{X} \rightarrow \mathrm{Y}) / \operatorname{supp}(\mathrm{Y}) .
$$

The previous measures of support, trust and elevation, are to obtain valid association rules, these measures to evaluate the quality of association rules and the elimination of data. These measures are also interesting calls, In general, they are used as input parameters by algorithms of induction of association rules [17].

\section{Apriori Algorithm}

The Apriori algorithm is a simple and popular association rules learning algorithm; its operation is based on the search of the sets of items with certain coverage. For this, first, the sets of formed by only one item that exceed the minimum coverage are built. This set of sets is used to construct the set of sets of two items, and so on until a size is reached in which there are no sets of items with the required coverage. The learning of association rules is commonly divided the phase of extraction of sets of items that meet the required coverage from the data, and the generation of the rules from these sets [3]. 
The Apriori algorithm is described below, which is based on all the observations presented above. The purpose of this algorithm is to efficiently find the frequent sets of items, for which it is proposed to intelligently apply the property of downward closure of the support that indicates that every subset $\mathrm{J}$ of a frequent set $\mathrm{I}$ is also frequent, likewise, if an itemset does not It is frequent, nor will the itemset that contain it. The specifications are the following: data: $\mathrm{T}$, transactions; entry: minsupp, minimum support threshold; variable: $C_{k}$, k-items candidates to be frequent; variable: $F_{k}$, k-frequent items; output: $\mathrm{U}^{\mathrm{k}}{ }_{\mathrm{i}=1} \mathrm{~F}_{\mathrm{i}}$, frequent sets of items [15].

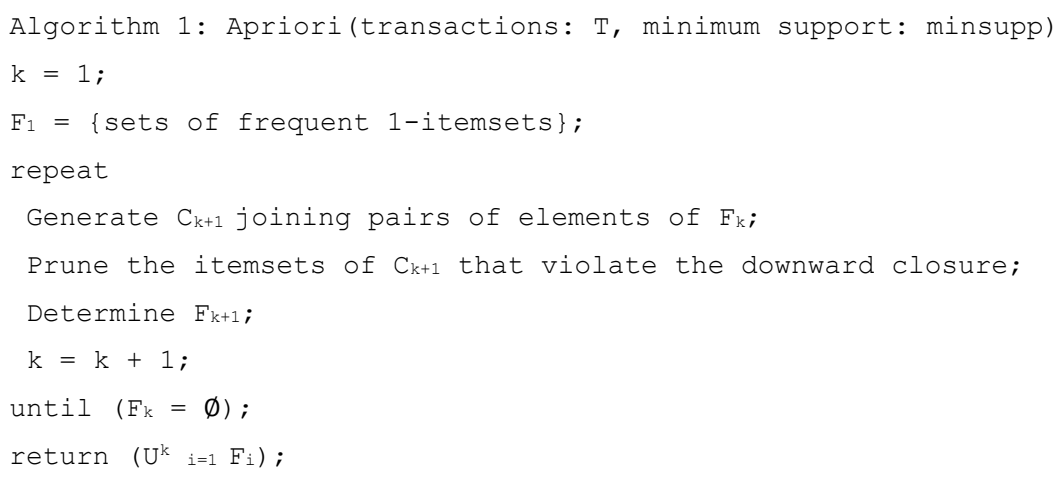

Algorithm 1. Apriori algorithm [15].

\section{Results}

A categorization was developed (see Table 1) to qualify the level of psychological engagement with the studies, where values were established that allow the evaluation of the observations of a case or group of cases [18]. There are other methods to calculate these levels through the UWES-S, to consult Schaufeli and Bakker [2].

Table 1. Categorization to qualify the UWES-S.

\begin{tabular}{lllll}
\hline Category & UWES-S & Vigor & Dedication & Absorption \\
\hline Very low & score $<2.20$ & score $<2.80$ & score $<2.50$ & score $<2.70$ \\
Low & $2.20 \leq$ score $<3.30$ & $2.80 \leq$ score $<3.80$ & $2.50 \leq$ score $<3.50$ & $2.70 \leq$ score $<3.60$ \\
Medium & $3.30 \leq$ score $<4.70$ & $3.80 \leq$ score $<5.20$ & $3.50 \leq$ score $<4.50$ & $3.60 \leq$ score $<4.70$ \\
High & $4.70 \leq$ score $\leq 6.00$ & $5.20 \leq$ score $\leq 6.00$ & $4.50 \leq$ score $\leq 6.00$ & $4.70 \leq$ score $<6.00$ \\
\hline
\end{tabular}

Source: Adapted from Schaufeli and Bakker [7]

The data set with which we worked for this study has 141 records of students from four careers in technologies: LSC, LIA, LTI and LT. The attributes that make up the data set are 16 in total, the values that make up this set of data are categorical, considering the nature of the type of study desired. Table 2 shows the correspondence between the values, as well as the scales to which they belong. 
Table 2. Correspondence between attributes and values, as well as their measurement scales. Source: Prepared by the researcher (2018).

\begin{tabular}{|c|c|c|c|}
\hline Attributes & Variable & Scale & Values \\
\hline Career & Carrera & Nominal & LSC/LIA,/LT/LTI \\
\hline Semester & Semestre & Nominal & $\begin{array}{l}\text { Tenth/Ninth/Eighth/Seventh/Sixth/Fifth/Fourth/ } \\
\text { Third/Second/ First. } \\
\text { Decimo/Noveno/Octavo/Septimo/Sexto/Quinto/Cuarto/ } \\
\text { Tercero/Segundo/Primero. }\end{array}$ \\
\hline Gender & Genero & Nominal & $\begin{array}{l}\text { Female/Male } \\
\text { Mujer/Hombre }\end{array}$ \\
\hline Age & Edad & Ordinal & $\begin{array}{l}\text { 22yGreater/21yLess22/18yLess21 } \\
\text { 22yMayores/21Menor22/ 18yMenor21 }\end{array}$ \\
\hline UWES-S & UWES-S & Ordinal & $\begin{array}{l}\text { VeryLow/Low /Medium/High } \\
\text { MuyBajo/Bajo/Medio/Alto }\end{array}$ \\
\hline Vigor & Vigor & Ordinal & $\begin{array}{l}\text { VeryLow/Low /Medium/High } \\
\text { MuyBajo/Bajo/Medio/Alto }\end{array}$ \\
\hline Dedication & Dedicación & Ordinal & $\begin{array}{l}\text { VeryLow/Low /Medium/High } \\
\text { MuyBajo/Bajo/Medio/Alto }\end{array}$ \\
\hline Absorption & Absorción & Ordinal & $\begin{array}{l}\text { VeryLow/Low /Medium/High } \\
\text { MuyBajo/Bajo/Medio/Alto }\end{array}$ \\
\hline Average & Promedio & Ordinal & $\begin{array}{l}\text { VeryLow/Low /Medium/High } \\
\text { MuyBajo/Bajo/Medio/Alto }\end{array}$ \\
\hline Selected career & Carrera.seleccionada & Nominal & $\begin{array}{l}\text { Yes/No } \\
\text { Si/No }\end{array}$ \\
\hline Failed class & Reprobado. materia & Nominal & $\begin{array}{l}\text { Yes/No } \\
\text { Si/No }\end{array}$ \\
\hline Change career & Cambio.carrera & Ordinal & $\begin{array}{l}\text { Nothing/VeryLittle/Little/Moderately/Much/Enough } \\
\text { Nada/ Muypoco/Poco/Medianamente/Mucho/Bastante }\end{array}$ \\
\hline Leaving university & Baja. universidad & Ordinal & $\begin{array}{l}\text { Nothing/VeryLittle/Little/Moderately/Much/Enough } \\
\text { Nada/ Muypoco/Poco/Medianamente/Mucho/Bastante }\end{array}$ \\
\hline $\begin{array}{l}\text { Finish successful } \\
\text { studies }\end{array}$ & Finalizar.exito & Ordinal & $\begin{array}{l}\text { Nothing/VeryLittle/Little/Moderately/Much/Enough } \\
\text { Nada/ Muypoco/Poco/Medianamente/Mucho/Bastante }\end{array}$ \\
\hline Financing & Financiamiento & Ordinal & $\begin{array}{l}\text { Parents/Work/Other } \\
\text { Padres/Trabajo/Otros }\end{array}$ \\
\hline Social class & Estrato.social & Ordinal & $\begin{array}{l}\text { LowLow/LowHigh/MediumLow/MediumHigh/ } \\
\text { HighLow/High High } \\
\text { BajaBaja/BajaAlta/MediaBaja/MediaAlta/AltaBaja/AltaAlta }\end{array}$ \\
\hline
\end{tabular}

Note. The variables and values of the data set are in Spanish, so its translation is shown in this table.

The Table 2 is used for the tests to be approved. The attributes career, semester, gender, identify the groups of students, for the values that take the dimensions that make up the UWES-S, that is, vigor, dedication, absorption, the categorization presented in Table 1 was considered. We considered the variables to complete the study, 
which imply in the decision of the stay and the continuity of the studies, as the average, others that can be registered as if they had found in the career they selected at the beginning of their studies, if have failed a class, if they have thought about changing careers or even leaving the university, if what they want is to finish their studies successfully, they will finish the two things about the studies of another school and another about the social stratum in which they find themselves.

\subsection{Association Rules}

When applying the algorithm A priori, using the arules package [19] of the R language. For the generation of the rules, the most common validation measures were considered: support, trust and lift. Thus, a support (s) of at least 0.25 and a confidence (c) of at least 0.9 was specified, and a lift (1) of at least 1 , which resulted in 37 rules in total, as follows: describe the ten rules considered most relevant, accompanied each of these of the support, trust and count of the times the rule is correctly applied (co) and lift (1). In Table 2, the values for each of the attributes of the data set can be verified. The 10 rules of interest presented contribute to the knowledge generated, since the value of lift obtained in each of them is greater than 1 , which means that their associations are statistically dependent, there is an association between the elements involved.

Rule 1

$\{$ dedication $=$ high, change.career $=$ nothing $\} \Rightarrow$ leaving.university $=$ nothing $\}$ $\mathrm{s}=0.39 \mathrm{c}=0.93 \mathrm{co}=56, \mathrm{l}=1.41$

Rule 1 indicates that if a student's dedication is high and he has not thought about changing his career, then he does not think about dropping out of the university at all.

Rule 2

$\{$ dedication $=$ high, selected.career $=$ yes, change.carrera $=$ nothing $=>\{$ baja.universidad $=$ nothing $\} \mathrm{s}=0.34 \mathrm{c}=0.92 \mathrm{co}=49 \mathrm{l}=1.40$

Rule 2 indicates that if a student's dedication is high, his career was selected and he has not thought about changing his career, then he does not think about unsubscribing from the university.

\section{Rule 3}

$\{$ absorption $=$ high $\} \Rightarrow$ uwess $=$ high $\} \mathrm{s}=0.34 \mathrm{c}=0.94 \mathrm{co}=49 \mathrm{l}=2.25$

Rule 3 indicates that if the absorption in a student is high then the psychological engagement with the studies is also high.

\section{Rule 4}

$\{$ uwess $=$ high, financing $=$ parents $\} \Rightarrow\{$ dedication $=$ high $\} \mathrm{s}=0.29 \mathrm{c}=0.95 \mathrm{co}=42$ $1=1.49$

Rule 4 indicates that if the psychological connection in a student is high and the financing of their studies is on the part of their parents, then the dedication is high. 
Rule 5

$\{$ absorption $=$ high, selected. career $=$ yes $\} \Rightarrow$ uwess $=$ high $\} \mathrm{s}=0.26 \mathrm{c}=0.95 \mathrm{co}=38$ $1=1.52$

Rule 5 indicates that if the absorption in a student is high and his career was selected, then the psychological connection with the studies is high.

Rule 6

$\{$ absorption $=$ high, financing $=$ parents $\Rightarrow$ uwess $=$ high $\} \mathrm{s}=0-26 \mathrm{c}=0.94 \mathrm{co}=37$ $1=2.26$

Rule 6 indicates that if the absorption in a student is high and the financing of their studies is on the part of their parents, then the psychological connection with the studies is high.

Rule 7

$\{$ dedication $=$ high, absorption $=$ high, selected .career $=$ yes $\} \Rightarrow$ uwess $=$ high $\}$ $\mathrm{s}=0.26 \mathrm{c}=0.94 \mathrm{co}=37 \mathrm{l}=2.26$

Rule 7 indicates that if the dedication in a student is high and the absorption is also high, as well as that the career was selected, then the psychological engagement with the studies is high.

\section{Rule 8}

$\{$ change. carrera $=$ nothing, finish. successful. studies $=$ much $\} \Rightarrow$ leaving. university $=$ nothing $\} \mathrm{s}=0.28 \mathrm{c}=0.90 \mathrm{co}=40 \mathrm{l}=1.37$

Rule 8 indicates that if a student has not thought about changing careers and thinks that he will finish his studies successfully, he does not think about canceling his university career.

Rule 9

$\{$ age $=18 y$ Less 21$\} \Rightarrow$ financing $=$ parents $\} \mathrm{s}=0.27 \mathrm{c}=0.92 \mathrm{co}=39 \mathrm{l}=1.30$

Rule 9 indicates that if the student's age is over 18 and under 21, the financing of their studies is on the parents' side.

\section{Rule 10}

$\{$ social class $=$ lowhigh $\} \Rightarrow$ selected career. $=$ yes $\} \mathrm{s}=0.27 \mathrm{c}=0.90 \mathrm{co}=39 \mathrm{l}=1.20$

Rule 10 indicates that if the social stratum is low high, the career they studied has been the one selected.

\subsection{Interactive Visual Representation of the Association Rules}

The rules of association are very useful in practical applications, however the number of rules that are generated can be very large, since hundreds of them can be generated, so the review and analysis of them can be a very complex task, almost impossible. A convenient way to explore the rules is through its interactive graphical visualization, in this way it is possible to identify in a more simple way which rules are considered interesting. 
Determining interest in a rule is not a simple task, one rule may be interesting for one analyst but not another, so the interest in a rule is considered subjective, depends on the knowledge and interests of the analyst [20]. Through the generation of visual association rules it is possible to facilitate this rule selection task. For the development of interactive visual association rules, the arulesViz package [21] of R. was used.

Next, the rules generated in a visual manner are presented for this case study. Figure 1 shows the 37 resulting rules in a general way, through this graph it is not possible to interact, which makes it difficult to explore the rules. Figure 2 shows the same graphic with the interactive property, the pink colored nodes, refer to the 37 rules, where the size of these circles depends on the number of occurrences of that rule, the green nodes are shown on the labels, where the attribute and the value it takes are specified.

These nodes when joined by arrows and their directions, allow the identification and reading of the rules. The left part of the rule are the attributes (green nodes) that go in the direction of the rule (pink node), the right side of the rule is indicated by the arrow in the exit direction of the rule (pink node) towards some attribute (green node). The labels shown in these diagrams are in Spanish, since the data set is in this language, so you can consult Table 2 to check the correspondence of the data.

\section{Graph for 37 rules}

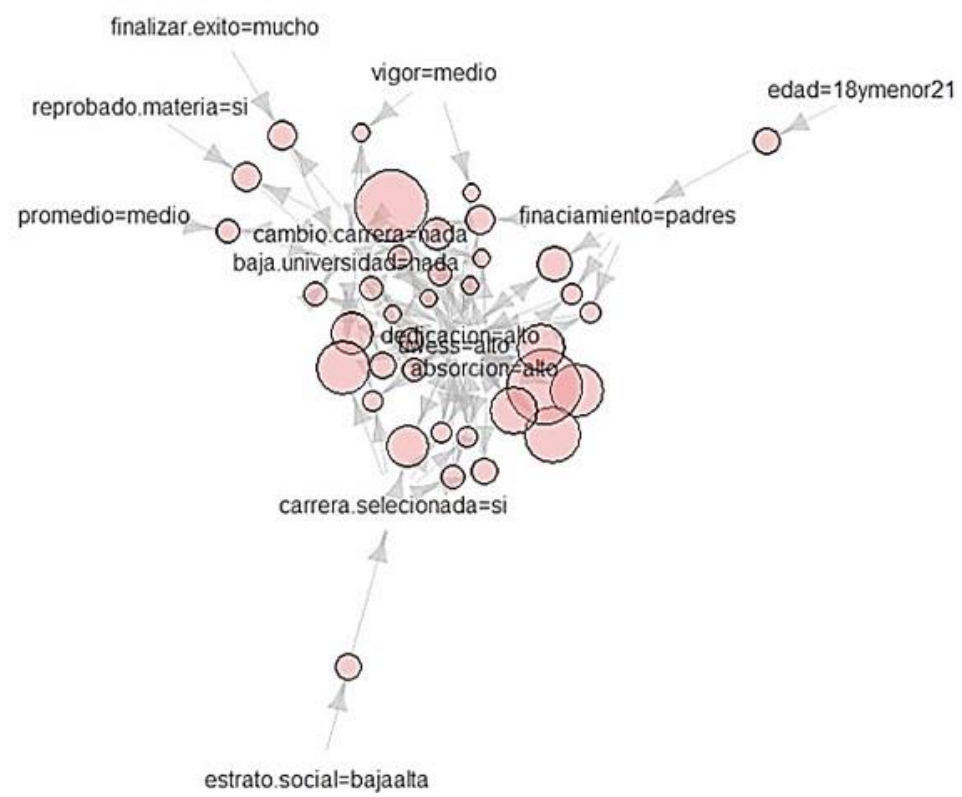

Fig. 1. Association Rules Chart. 
In Figure 1 where the general diagram is observed, it is in the lower left, the case of Rule 1, for a better description is presented Figure 3, where this rule is focused (indicated by the pink node) receives as input of two attributes (indicated by green nodes): dedication $=$ high and change . career $=$ nothing, resulting in a red dotted line, directed to the attribute leaving.university = nothing (green node). This Rule 1 indicates that if a student's dedication is high and he has not thought about changing his career, then he does not think about dropping out of the university at all. It is possible to manipulate the visualization of these rules and find the routes that the rule explains when selecting and moving the mouse by the different elements that make up the rules.

\section{Rule 1}

$\{$ dedication $=$ high, change carrera $=$ nothing $\} \Rightarrow>\{$ leaving university $=$ nothing $\}$

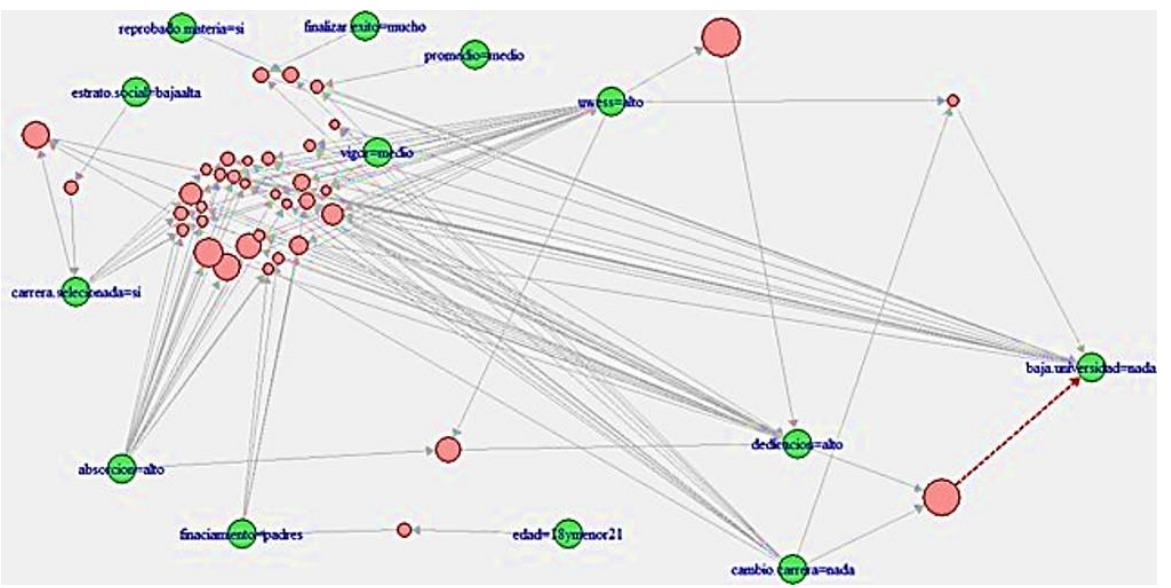

Fig. 2. Interactive Association Rules Chart.

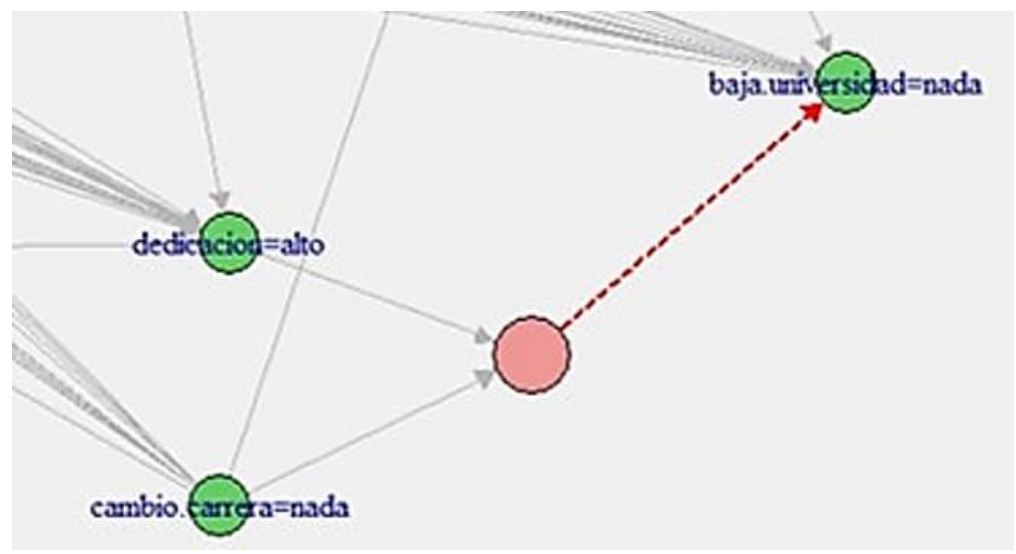

Fig. 3. Rule 1 through Visual Association. 
Another rule that is observed is Rule 9 (See Figure 4) where it is indicated that if the student's age is greater than 18 and less than 21, the financing of their studies is on the part of the parents:

Rule 9

$\{$ age $=18$ yLess 21$\} \Rightarrow$ financing $=$ parents $\}$

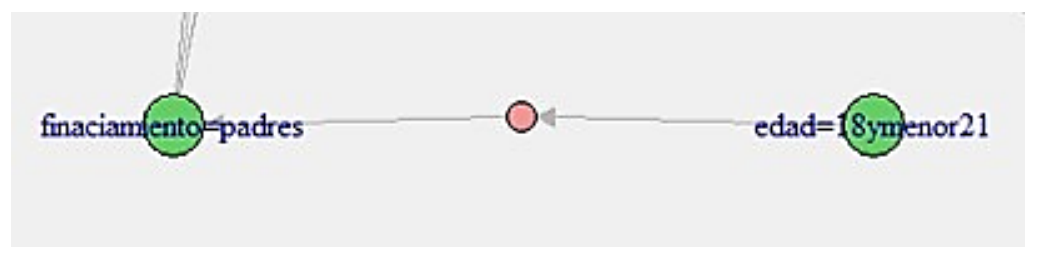

Fig. 4. Rule 9 through Visual Association.

The nodes that represent Rule 1 and Rule 9 (pink nodes) have different sizes (see Figure 3 and Figure 4), the node of Rule $1(\mathrm{co}=56)$ is larger than the node of Rule 9 $(c o=39)$, since it depends on the number of occurrences that the rule is fulfilled.

\section{Conclusions}

The association rules allow finding interesting results as a first approach to the data, since they help to solve data mining tasks of descriptive type. It is considered useful when the nature of the data is categorical, as is the case of the data generated by instruments such as questionnaires, surveys, among others. They allow the reading of the data in a more natural way with respect to other techniques, although when executing this type of algorithms for the generation of association rules, inconveniences such as the number of rules can be presented, since hundreds of algorithms can be generated.

The selection of the rules can be complicated for the analysts, due to the number of rules, besides that the election of the various rules of interest from one analyst to another, up to a certain point is subjective. One way to help a better selection of the rules of association is through its visual representation, and one step further its manipulation, that is, the unfolding of the rules interactively. In this study, this form of knowledge discovery was selected given the nature of the data.

The objective of this study was to find rules of association of interest to determine the relationship of the dimensions of the psychological connection or student engagement with other selected variables that have an impact on the decision of stay and continuity of the students.

This is an approach to this type of studies making use of this technique and it is intended to add more elements that allow knowing more about the psychological connection with the studies. The resulting rules indicate that the dedication variable is the one that has more presence in this connection with the studies to maintain and conclude successfully, that is, the meaning is important for their studies, feeling excited and proud of their career. 


\section{References}

1. Salanova, M., Schaufeli, W.B.: El Engagement de los empleados un reto emergente para la dirección de recursos humanos. Estudios Financieros 261, 109-138. http://www.wilmarschaufeli.nl/publications/Schaufeli/221.pdf (2004)

2. Schaufeli, W.B., Bakker, A.: Utrecht Work Engagement Scale (UWES). Escala de Engagement en el trabajo de Utrecht, Ocuppational Health Psychology Unit: Utrech University.http://www.wilmarschaufeli.nl/publications/Schaufeli/Test\%20Manuals/Test_manual_UWES_Espanol.pdf (2003)

3. Hernández, J., Ramírez, M.J., Ferri, C.: Introducción a la Minería de datos. Madrid, España: Prentice Hall (2008)

4. Correa, J.C.: Gráficos Estadísticos con R. Universidad Nacional-Sede Medellín (2002)

5. R Development Core Team: A language and environment for statistical computing. R Foundation for Statistical Computing, http://www.R-project.org (2016)

6. Reyes-Nava, A. Flores-Fuentes, A., Alejo, R., Rendón-Lara, E.: Minería de datos aplicada para la identificación de factores de riesgo en alumnos. Research in Computing Science 139, 177-189 (2017)

7. Rodríguez-Maya, N., Lara-Álvarez, C., May-Tzuc, O., Suárez-Carranza, B.A.: Modeling Student' Dropout in Mexican Universities. Research in Computing Science 139, 163175 (2017)

8. Márquez-Vera, C., Romero-Morales, C., Ventura-Soto, S.C., Ventura, S.: Predicción del Fracaso Escolar mediante Técnicas de Minería de Datos. IEEE-RITA 7(3) (2012)

9. González-Marrón, D., Enciso-Gonzalez, A., Hernandez-Gonzalez, A.K., Gutierrez-Franco, D., Guizar-Barrera, B., Marquez-Callejas, A.: Evaluación de parámetros de encuesta de ingreso del CENEVAL para alumnos candidatos a ingresar al nivel superior, caso de estudio ITP. Research in Computing Science 139, 135-147 (2017)

10. Ivkovic, S., Yearwood, J., Stranieri, A.: Visualizing Association Rules for feedback within the legal system. ACM 1-58113-747-8. Edinburgh, Scotland, UK (2003)

11. Lee, J., Han, J., Chi, K.: Mining Quantitative Association Rule of Earthquake Data. In: International Conference on Convergence and Hybrid Information Technology. Daejeon, Korea (2009)

12. Morales-Mateos, E.Y., Hernández-Aguilar, J.A., Ochoa-Ortíz Zezzatti, C.A., López Garrido M.A.: A Comparison Represented in the Form of Radar of University Student Engagement in Degrees in Technologies. Research in Computing Science 122, 141-151 (2016)

13. Lopez-Garrido, M.A., Hernández-Aguilar, J.A., Ochoa-Ortiz Zezzatti, C.A., MoralesMateos, E.Y., González-Constantino, C.: Comparative Study of Learning Strategies of Bachelor Students in Nursing. Research in Computing Science 122, 153-162 (2016)

14. Guisande, C., Vaamonde, A.: Gráficos estadísticos y mapas con R. España: Ediciones Díaz de Santos (2013)

15. Exposito, C., Exposito, A., López, I., Melián, B., Moreno, J.: Minería de patrones de asociación. Departamento de Ingeniería Informática y de Sistemas. Universidad de La Laguna, https://campusvirtual.ull.es (2018)

16. Haro, V., Péres, W., Saquicela, V.: Bibliomining para descubrir reglas de asociación en el Centro de Documentación Regional "Juan Bautista Vázquez". Departamento de Ciencias de la Computación, Universidad de Cuenca, http://dspace.ucuenca.edu.ec/bitstream/123456789/26350/1/TICEC_2016_20.pdf (2016)

17. Pincho, J.: Métodos de clasificación basados en asociación aplicados a sistemas de recomendación. Universidad de Salamanca, https://gredos.usal.es/jspui/bitstream/10366/83342/1/DIA_PinhoLucasJ_Métodosdeclasificación.pdf (2010) 
Erika Yunuen Morales Mateos, María Arely López Garrido, José Alberto Hernández Aguilar, et al.

18. Cuñer, N.: Escala de Inteligencia para Niños de Wechsler, WISC-IV. Diccionario de Psicome-tría. Montevideo, Uruguay, http://psicologos.org.uy/documentos13/20130926_Diccionario\%20de\%20psicometria.pdf (2013)

19. Hahsler, C., Buchta, C., Gruen, B., Hornik., K., Johnson, I., Borgelt, C.: Package 'arules'. https://cran.r-project.org/web/packages/arules/arules.pdf (2018)

20. Liu, B., Hsu, W., Wang, K., Chen, S.: Visually Aided Exploration of Interesting Association Rules. In: Zhong N., Zhou L. (eds), Methodologies for Knowledge Discovery and Data Mining. PAKDD 1999. Lecture Notes in Computer Science, vol. 1574. Springer, Berlin, Heidelberg (1999)

21. Hahsler, C., Tyler, G., Chelluboina, S.: Package 'arulesViz'. https://cran.r-project.org/web/packages/arulesViz/arulesViz.pdf (2018) 